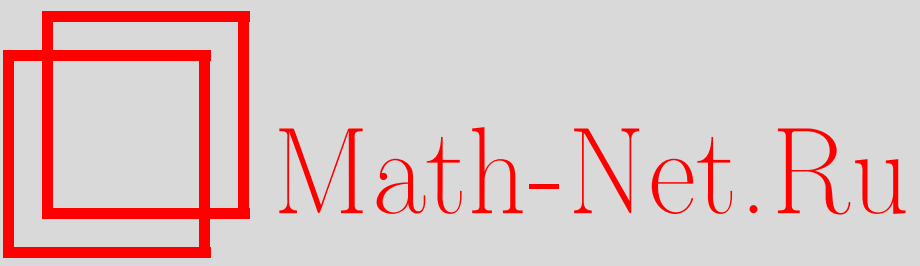

Д. Н. Иванов, Сбалансированные базисы инволютивного типа, УМН, 2004, том 59, выпуск 3, 169-170

DOI: https://doi.org/10.4213/rm748

Использование Общероссийского математического портала Math-Net.Ru подразумевает, что вы прочитали и согласны с пользовательским соглашением

http://www.mathnet.ru/rus/agreement

Параметры загрузки:

IP : 54.196 .121 .252

26 апреля 2023 г., 14:17:08 


\section{СБАЛАНСИРОВАННЫЕ БАЗИСЫ ИНВОЛЮТИВНОГО ТИПА}

\section{Д.Н. ИвАнов}

1. В статье [1] приводится конструкция сбалансированных базисов в алгебрах матриц нечетного примарного порядка. Она опирается на существование в таких алгебрах ортогонального базиса из инволюций с одинаковым следом. В данной статье показьвается, как использовать это обстоятельство для построени сбалансированных базисов в алгебрах матриц нечетного порядка. Кроме этого, обобщается известная теорема, утверждающая, что порядок регулярной матрицы Адамара ${ }^{1}$ равен квадрату суммы элементов ее строки.

2. Напомним основное определение, которое впервые появилось в работе [2]. Всюду далее под алгебр ой понимается ассоциативная полупростая конечномерная над полем комплексных чисел $\mathbb{C}$ алгебра, т.е. то, что обьчно назьвают классически полупростой алгеброй над $\mathbb{C}$. Обозначим через $\operatorname{Tr}_{A}=\operatorname{Tr}$ след регулярного (правого или левого) представления алгебры $\mathbf{A}, \operatorname{Tr}_{A} x$ будем называть регулярным следом элемента $x \in \mathbf{A}$; как обычно, следом матрицы $A$ будем называть сумму ее диагональных элементов и обозначать $\operatorname{tr} A ; \mathbf{1}_{A}-$ единичньй элемент в $\mathbf{A}$. Элементы $x, y \in \mathbf{A}$ будем назьвать ортогональныли, если $\operatorname{Tr}_{A} x y=0$.

ОПредЕлениЕ 1. Семейство идемпотентов $\mathscr{E}=\left\{e_{1}, \ldots, e_{v}\right\}$ в алгебре $\mathbf{A}$ (здесь $\operatorname{dim} \mathbf{A}>1$ ) называется сбалансированной $t$-системой, если выполнены следующие два условия:

(1) (условие сбалансированности) $e_{1}+\cdots+e_{v}=k \cdot \mathbf{1}_{A}, k \in \mathbb{C}$;

(2) (условие равноугольности) для любых $s$ различных идемпотентов $e_{i_{1}}, \ldots, e_{i_{s}} \in \mathscr{E}$, где $s \leqslant t$, значение функции следа

$$
\operatorname{Tr}_{A} e_{i_{1}} \cdots e_{i_{s}}=\lambda_{s}
$$

зависит только от $s$.

Если сбалансированная 2-система $\mathscr{E}$ является базисом алгебры $\mathbf{A}$, то $\mathscr{E}$ называется сбалансированным базисом, а $\mathbf{A}$ назьвается сбалансированной алгеброй.

Лемма 1. Пусть попарно ортогональные инволюиии $\left\{\sigma_{i}, i \in I\right\}$ с одинаковым регулярньм следом образуют базис алгебры А. Тогда семейства идемпотентов Еீ' $=$ $\left\{\left(\mathbf{1}_{A}+\sigma_{i}\right) / 2, i \in I\right\}$ и $\mathscr{E}^{-}=\left\{\left(\mathbf{1}_{A}-\sigma_{i}\right) / 2, i \in I\right\}$ являются сбалансированными базисами.

ОПРЕдЕЛЕниЕ 2. Семейства идемпотентов $\mathscr{E}^{ \pm}$, о которых идет речь в лемме 1 , назьваются сбалансированными базисами инволютивного типа.

Отметим, что если $\mathbf{A}$ - коммутативная алгебра размерности $n$, то $\mathbf{A}$ допускает сбалансированньй базис инволютивного типа тогда и только тогда, когда существует регулярная матрица Адамара порядка $n$. Хорошо известно, что в этом случае $n$ должно быть квадратом четного числа, равного сумме элементов строки матрицы Адамара. Предполагается, что при всех таких $n$ регулярные матрицы Адамара порядка $n$ существуют.

В статье [1] приводится следующая конструкция сбалансированных базисов инволютивного типа в алгебрах матриц нечетного примарного порядка.

Определим $(p \times p)$-матрицы $D, P$ и $J_{a, b}$ :

$$
\begin{gathered}
D=\left(\begin{array}{ccccc}
1 & & & & 0 \\
& \varepsilon & & & \\
& & \varepsilon^{2} & & \\
& & & \ddots & \\
0 & & & \varepsilon^{p-1}
\end{array}\right), \quad P=\left(\begin{array}{ccccc}
0 & 0 & \ldots & 0 & 1 \\
1 & 0 & \ldots & 0 & 0 \\
0 & 1 & \ldots & 0 & 0 \\
\ldots & \ldots & \ldots & \ldots & \\
0 & 0 & \ldots & 0 & 1
\end{array}\right), \\
J_{a, b}=D^{a} P^{b},
\end{gathered}
$$

Работа выполнена при поддержке Российского фонда фундаменталњных исследований (грант № 02-01-00219)

${ }^{1}$ Под регулярной матрицей Адамара $H$ понимается квадратная матрица с попарно ортогональными строками, состоящая из \pm 1 и такая, что сумма элементов каждой строки в $H$ постоянна.

(C) Д.Н. Иванов 2004 
где $\varepsilon$ - примитивньй корень степени $p$ из 1 . Удобно рассматривать $a$ и $b$ как элементы поля вычетов $\mathbb{F}_{p}=\mathbb{Z} / p \mathbb{Z}$. $\mathrm{C}$ каждым вектором $u=\left(a_{1}, \ldots, a_{n} ; b_{1}, \ldots, b_{n}\right) \in V=\mathbb{F}_{p}^{2 n}$ свяжем матрицу $J_{u}=J_{a_{1}, b_{1}} \otimes \cdots \otimes J_{a_{n}, b_{n}}$. Пусть $u^{\prime}=\left(a_{1}^{\prime}, \ldots, a_{n}^{\prime} ; b_{1}^{\prime}, \ldots, b_{n}^{\prime}\right) \in \mathbb{F}_{p}^{2 n}$. Тогда $J_{u} J_{u^{\prime}}=\varepsilon^{B\left(u, u^{\prime}\right)} J_{u+u^{\prime}}$, где $B\left(u, u^{\prime}\right)=-\sum_{i=1}^{n} a_{i}^{\prime} b_{i} ; J_{u} J_{u^{\prime}} J_{u}^{-1}=\varepsilon^{\left\langle u \mid u^{\prime}\right\rangle} J_{u^{\prime}}$, где $\left\langle u \mid u^{\prime}\right\rangle=\sum_{i=1}^{n}\left(a_{i} b_{i}^{\prime}-a_{i}^{\prime} b_{i}\right)=B\left(u, u^{\prime}\right)-B\left(u^{\prime}, u\right)$.

ПредложениЕ 1 [1]. Инволютивные матриць

$$
A_{x}=\frac{1}{p^{n}} \sum_{u} \varepsilon^{\langle u \mid x\rangle+\frac{1}{2} B(u, u)} J_{u}, \quad x \in \mathbb{F}_{p}^{2 n},
$$

где суммирование ведется по всем $u=\left(a_{1}, \ldots, a_{n} ; b_{1}, \ldots, b_{n}\right) \in \mathbb{F}_{p}^{2 n}$, имеют одинаковый регулярный след $\left(\operatorname{Tr} A_{x}=p^{n} \operatorname{tr} A_{x}=p^{n}\right)$ и образуют ортогональный базис в алгебре $\mathrm{M}_{p^{n}}(\mathbb{C}), p$ - нечетное простое.

Лемма 2. Пусть в алгебрах $\mathbf{A}$ и $\mathbf{B}$ существуют ортогональные базисы из инволюций с одинаковым регулярнылм следом: $\mathbf{A}=\left\langle A_{i}, i \in I\right\rangle, \mathbf{B}=\left\langle B_{j}, j \in J\right\rangle, \operatorname{Tr}_{A} A_{i}=r_{a}$, $\operatorname{Tr}_{B} B_{j}=r_{b}$. Тогда инволючии $\left\{A_{i} \otimes B_{j}, i \in I, j \in J\right\}$ образуют ортогональный базис алгебры $\mathbf{A} \otimes \mathbf{B}$ и имеют одинаковыи ие реглярныи след $\operatorname{Tr}_{A \otimes B} A_{i} \otimes B_{j}=r_{a} r_{b}$.

ТЕОРема 1. Пусть попарно ортогональные инволючии $\sigma_{1}, \ldots, \sigma_{n}$ с одинаковым регулярным следом $r$ образуют базис алгебры $\mathbf{A}$. Тогда $n=r^{2}$.

ДоКАЗАТЕЛЬСтво. Разлагая единичный элемент $\mathbf{1}_{A}$ по базису $\left\{\sigma_{i}\right\}$ и используя ортогональность инволюций, выводим

$\mathbf{1}_{A}=\sum_{i=1}^{n} c_{i} \cdot \sigma_{i} \Rightarrow r=\operatorname{Tr}\left(\mathbf{1}_{A} \cdot \sigma_{j}\right)=\sum_{i=1}^{n} c_{i} \cdot \operatorname{Tr}\left(\sigma_{i} \sigma_{j}\right)=c_{j} \cdot \operatorname{Tr} \sigma_{j}^{2}=c_{j} \cdot \operatorname{Tr} \mathbf{1}_{A}=c_{j} n \Rightarrow c_{j}=\frac{r}{n}$.

Далее, применяя к обеим частям равенства $\mathbf{1}_{A}=(r / n) \sum_{i=1}^{n} \sigma_{i}$ функцию $\mathrm{Tr}$, получаем $n=$ $(r / n) \cdot r n$. Значит, $n=r^{2}$. Теорема доказана.

Теорема 2. Алгебра матрич $\mathrm{M}_{n}(\mathbb{C})$ тогда и только тогда допускает сбалансированный базис инволютивного типа, когда $n$ нечетно.

ДокАЗАТЕльСтво. Так как $\mathrm{M}_{k}(\mathbb{C}) \otimes \mathrm{M}_{l}(\mathbb{C}) \cong \mathrm{M}_{k l}(\mathbb{C})$, то существование сбалансированного базиса инволютивного типа в алгебре матриц $\mathrm{M}_{n}(\mathbb{C})$, где $n$ нечетно, вытекает из предложения 1 и леммы 2.

Обратно, если инволютивные матрицы $A_{i}$ с одинаковым регулярным следом $\operatorname{Tr} A_{i}=r$ образуют ортогональный базис алгебры $\mathrm{M}_{n}(\mathbb{C})$, то в силу теоремы $1 r=n$. Значит, $\operatorname{tr} A_{i}=$ $(1 / n) \operatorname{Tr} A_{i}=1$. Но след инволютивной матрицы имеет ту же четность, что и ее порядок. Поэтому $n$ нечетно. Теорема доказана.

Отметим, что алгебра $\mathrm{M}_{2}(\mathbb{C})$ сбалансирована (см. [2]), но кроме нее пока не известно ни одной сбалансированной алгебры матриц четного порядка.

Автор благодарен проф. М.В.Зайцеву и проф. А. В. Михалеву за внимание к работе.

\section{СПИСОК ЛИТЕРАТУРЫ}

[1] Д. Н. Иванов // Матем. сб. 2001. Т. 192. № 4. С. 73-86. [2] Д. Н. Иванов // Матем. сб. 1998. Т. 189. № 12. С. $83-102$. 